\title{
Credibility Evaluation of Simulation Models
}

\author{
LIN Qi ${ }^{1, a}, \mathrm{LI} \mathrm{ZHI}^{1, b}$ \\ ${ }^{1}$ Department of Space and Command, Academy of Equipment, Beijing, China \\ aLinqi_98@163.com, 'bizhi@163.com
}

Keywords: Credibility Evaluation; Simulation model; Verification; Validation

\begin{abstract}
This paper discusses an evaluation methodology that can be used to assess the credibility of simulation models. The goal of verification and validation of simulation models will be described, together with a description of evaluation methodology and evaluation procedures. The credibility of simulation models can be evaluated using the method proposed here, which will be much meaningful to the simulation systems development. Taking a space Tether-net simulation system as an instance, the credibility evaluation result is provided.
\end{abstract}

\section{Introduction}

Evaluation is the key in making significant progress in applying solutions and algorithms to real life problems and simulation systems. With the development of the simulation technology, there is a great need for the simulation of the complex system. Especially as the abroad use of the large-scale complex distributed system, the comparability of the simulation system compared with the prototype system has held the attention of the researchers.

The effectiveness and the performance of any developed simulation model will be determined by means of evaluation, verification and validation. However, systematic ways to evaluate how different simulation models work and whether they can be trusted and applicable are needed, as well as ways of evaluating credibility based on practical experiments using useful collected data.

\section{Goal of verification and validation}

As concerning verification and validation, the key is to build the model right, which means to compare the conceptual model to the computer representation, so as to answer such questions as follows:

- Is the model implemented correctly in the computer?

- $\quad$ Are the input parameters and logical structure of the model correctly represented?

- Is the model an accurate representation of the real system?

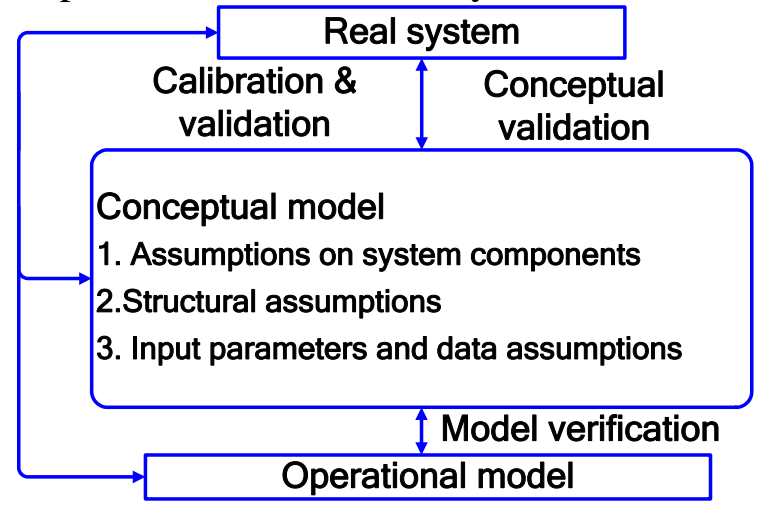

Figure 1 Verification and Validation of Simulation Models

As shown, validation is the overall process of comparing the model and its behavior to the real system and its behavior. Calibration is the iterative process of comparing the model to the real system. The purpose of verification and validation of simulation model is to assure that the conceptual model is represented accurately in the computerized representation, and to determine whether the 
conceptual model accurately represented by the operational model, which generally has assumptions on system components, system structure, parameter values, abstractions, and simplifications. For example, Data assumptions should be based on the collection of reliable data and correct statistical analysis.

In fact, a simulation model can be viewed as an input-output transformation. It accepts values of the input parameters and transforms these inputs into output. It is necessary to find the appropriate techniques both to assure model accuracy and to assure model credibility.

\section{Evaluation Methodology}

The evaluation methodology should be based upon the reliability and precision of collected data, as well as a characterized scheme using simulation techniques. The evaluation methodology should fulfill the requirement of developing an accurate, useful procedure of evaluation of the proposed techniques which could be used in the future. This will provide valid methods for comparing results obtained from the experiments and/or other similar development work.

Validation determines whether a simulation model is an acceptable representation of a real system given the purpose of a simulation model. If the purpose of a model is to answer particular questions, the validity of the model has to be determined with respect to each question. There are many validation techniques that can be used to validate a particular model. These techniques include Parameter Variability-Sensitivity Analysis, Predictive Validation, Operational Graphics, Historical Data Validation, Historical Methods, Multistage Validation, Traces, Turing Tests, and so on.

However, these are general purpose techniques ${ }^{[1]}$. With regard to simulation system with special mission, it should be analyzed from the aspects of overall analysis and structure analysis. The item of the structure analysis is to acquire the quantitative credibility result of the models on the view of microcosmic analysis; and the goal of the overall mission is to gain the qualitative representation on the view of microscopically analysis, the approach divides the mission into a series of mission sections, every section is composed of a group of actions.

The method has several advantages. First, it is easy to practice, and the analysis is simple. Second, it forms different dynamic methods of credibility evaluation for different mission with the same system, it is more reasonable in logic, and enforce the usability of the credibility evaluation model, compared with the static methods. And last, through the sort of the microcosmic analysis, it descends the infection that the model modification and branch make on credibility evaluation work.

\section{Credibility Evaluation Procedure}

Credibility evaluation based on action. Through the analysis of the system, it is clear that every action has different function in the model. For the different of the function that the actions performs, the credibility evaluation viewpoints should be different, as it may focus on the maneuverability of the action on one action and the other may the dependability, and the corresponding evaluation work will adjusts to the indexes and the requirement, for the work will be reduced or suspended if the index is unimportant or out of requirement, after analysis the importance of the index, the index system will be established.

For every index, the evaluation method may be different, as the mature technology will be adopted here, or make a innovation aim at the different inherent characteristic of the index, but no mater which method, the processes of the evaluation on the logic will be similar. So, here the model using the IDEF0 method is performed as the Fig. 2.

Input: means the data, resource, and experience will be transformed in the process of action credibility evaluation; Control: means the conditions limited in the process of action credibility evaluation; Mechanism: means the personnel and the resources will present of the transform but will be absorbed in the credibility evaluation; Output: means the quantitative results of the index, names the products of the credibility evaluation. 


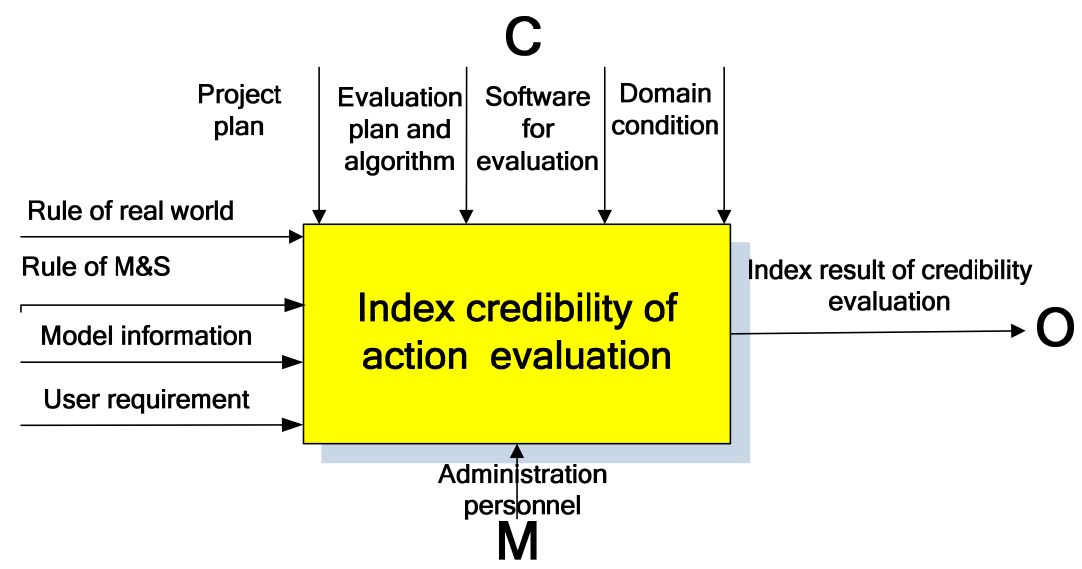

Figure.2 index credibility of action evaluation

Fig.1 points out four aspects of the action credibility evaluation: Output contains the overall user requirements, the principles of the prototype system, the principles which should be kept in the process of the modeling and simulation and the information of the system, here the system information includes configuration information of the model establishment and execution, the experience and the external data that can be acquired; Control, means the guidance and the condition of the simulation plan and evaluation plan that will play on the process of modeling and simulation, that should be supported by specific evaluation software and tools; Mechanism, prescribes the evaluation personnel, quantitative description, actually, that also could be standardized documents, the reason to use quantitative approach is to get a intuitive impression of the evaluation work.

Based on the evaluation results acquired in the upside, the mathematic model will be established (multiple differentiate model, PHAP, expert evaluation method and so on, there is no rule for the methods, several methods can integrated to solve the question, the only standard is to resolve the question logically and availably), and the quantitative results will be calculated.

Credibility evaluation based on the division of mission. For the different simulation mission, it can be divided up a series of actions or a chain of action groups. In the process of the simulation, based on the action as one cell, the mission can be cut into pieces, every section plane has a series of actions which are exhibited on time sequence, for there are $n$ actions, in one period of time, several action will be operated to be an action group. Anyway, the aim of the phase is to establish the action time sequence from the division of the mission.

Based on the time sequence of action established in the forepart, the mathematic model of action time sequence can be founded (such as Petri net, exception tree and so on). Through the mathematic model of action time sequence, the mathematic model of credibility evaluation will be established, and will acquire the final result by calculating. Here it needs to specific represent the credibility evaluation process, one of characteristics of credibility evaluation is agile, this paper evaluates the action credibility, then introduces the method of the mission credibility evaluation, as to acquire the mission credibility evaluation result, it can also adopt other methods, for examples, for a single index, the index of mission credibility evaluation will be sure based the action credibility evaluation, it performs certainly that with different methods, different credibility evaluation results will be acquired in content and form.

\section{Instance Analysis}

Here take a space Tether-net simulation system as an example to illuminate the application of the approach. Tether net can accomplish in-orbit service, space garbage, some military things and significant dual task. At present outside space powers carry out research plan, such as American FREND $^{[3]}$ and Germanic ESS ${ }^{[4]}$. This shows that it is difficult to resolve many of the key technologies, include objective measurement of space, close to the dock technology, arresting agency technology, contact-impact dynamics technology, combination and stable technology and so on. So it is very important to validate the performance and efficiency of tether net system by simulation and testing. 
On the basis of analyzing and concluding the traditional test technology using simulation-based testing methods ${ }^{[5-7]}$, the basic configuration of tether net system operating environment is designed, shown in Fig. 3.

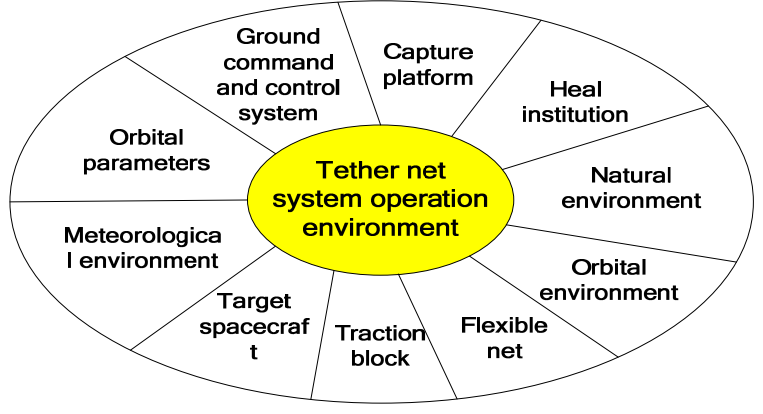

Fig.3 basic configuration of tether net system operating environment

The system is composed of 6 models, every model can complete an action, the scenario sets before the execution, and the mission can be divided into actions pieces. The actions index system is established as Table 1.

Table 1 the actions index system

\begin{tabular}{|l|l|l|l|l|l|l|}
\hline model & A & B & C & D & E & F \\
\hline \multirow{4}{*}{ index } & integrality & integrality & integrality & integrality & integrality & integrality \\
\cline { 2 - 7 } & consistency & consistency & consistency & consistency & consistency & consistency \\
\cline { 2 - 7 } & traceability & veracity & veracity & traceability & veracity & traceability \\
\cline { 2 - 7 } & veracity & Interoperation & Interoperation & veracity & reliability & Interoperation \\
\cline { 2 - 7 } & reliability & compatibility & reliability & compatibility & & compatibility \\
\cline { 2 - 7 } & Interoperation & & & & & \\
\hline
\end{tabular}

It establishes mission credibility model of system considering maintenance support and spares supply, finally, the mission credibility evaluation result is $\mathrm{D}=0.822$.

\section{Inclusion}

This paper points out a method to resolve the credibility evaluation problem of the simulation system based on mission. The credibility of simulation models can be evaluated using the method proposed here, which will be much meaningful to the simulation systems development. Taking a space Tether-net simulation system as an instance, the credibility evaluation result is provided. However, it lack optimization research, which will be the main work in the further.

\section{References}

[1] Sun Shixia, Research on Credibility Evaluation of Modeling and Simulation for Large-Scale Complex System. Changsha: Graduate School of National University of Defense Technology. 2005.

[2] Carzaniga A, Rosenblum DS, WolfAL. Design and evaluation of a wide-area event notification service[J]. ACM Trans. Computer Systems, 2001, 19(3), P332-383.

[3] DARPA Tactical Technology Office. Front-end robotic enabling near-term demonstrations(FREND)Project Page. [2010-04-03 ].http://www.Darpa.mil/tto/ programs/frend.htm.

[4] DLR.ESS. http:/www.dlr.de/rm/ en/desktopdefault.aspx/tabid-3827/5969-read-8750/.

[5] US DoD, SBA Task Force. SBA Roadmap . http:// www.msosa.dmso.mil/sba_documents/, 1998

[6] Hoyt R P, Slostad J T,Frank S S. A Modular Momentum- exchange /Electroodynamic-reboost Tether System Architecture.ALAA2003-5214,2003

[7] I Jose Sepulveda, Luis Rabelo, aebok Park,Frank Riddick. Implementing the High Level Architecture in the Virtual Test Bed.Proceedings of the 2004 Winter Simulation Conference. 2004: 1444-1451. 